\title{
Deformation localization in metallic glasses studied by in situ TEM deformation
}

\author{
Christoph Gammer ${ }^{1}$, Thomas C. Pekin ${ }^{2,3}$, Colin Ophus ${ }^{2}$, Andrew M. Minor ${ }^{2,3}$, Jürgen Eckert ${ }^{1,4}$ \\ ${ }^{1}$ Erich Schmid Institute of Materials Science, Austrian Academy of Sciences, Jahnstraße 12, 8700 \\ Leoben, Austria. \\ ${ }^{2}$ National Center for Electron Microscopy, Molecular Foundry, Lawrence Berkeley National \\ Laboratory, Berkeley, California 94720, USA. \\ ${ }^{3}$ Department of Materials Science and Engineering, University of California, Berkeley, California \\ 94720, USA. \\ ${ }^{4}$ Department Materials Physics, Montanuniversität Leoben, Jahnstraße 12, 8700 Leoben, Austria.
}

Metallic glasses (MGs) are an exciting class of materials due to their unique properties, such as high strength and good wear resistance [1]. A major drawback is their limited ductility caused by the formation of shear bands leading to catastrophic failure. While progress in the understanding of early stages of deformation localization was made using colloidal solids or molecular dynamics simulations [2], an experimental understanding is still lacking. The reason is the disordered nature of MGs inhibiting direct imaging of the fundamental deformation mechanisms. Therefore, we use in situ deformation in the transmission electron microscope (TEM) to study their deformation at the nanoscale. Rod-shaped $\mathrm{CuZrAlAg} \mathrm{MG} \mathrm{specimens} \mathrm{are} \mathrm{produced} \mathrm{by} \mathrm{suction-casting} \mathrm{and} \mathrm{samples} \mathrm{for} \mathrm{in} \mathrm{situ} \mathrm{deformation} \mathrm{are} \mathrm{made}$ by focused ion beam machining. Deformation is carried out under displacement control using a Hysitron PI-95 Picoindenter. In addition to standard compression tests of pillars, tension tests are carried out.

Figure 1 shows the result from an in situ tensile test of a $200 \mathrm{~nm}$ thick specimen acquired in TEM bright-field mode. The load-displacement curve shows elastic deformation followed by abrupt fracture. Figure 1a-c shows three frames corresponding to the initial state, the elongated state and the sample after fracture, demonstrating fracture along a single shear plane. To study the effect of the structure and size of the MGs on their mechanical properties, in situ deformation is carried out on different specimens using the same experimental setup. In comparison to the $200 \mathrm{~nm}$ thick specimen, the tensile test of a $65 \mathrm{~nm}$ thick specimen shows deformation localization without fracture resulting in extended ductility and necking. The ductility in nanosized specimens is caused by a size effect observed for MGs [3]. Finally, deformation of MG composites containing crystals formed through thermal treatment shows the occurrence of multiple shear bands. The comparison of these tests demonstrates that the structure and size strongly influences the deformation localization, and that this localization subsequently determines the overall mechanical properties of the MGs.

The knowledge of the local transient strain field during deformation is required to investigate early stages of deformation localization, but conventional strain measurements based on X-ray diffraction do not provide sufficient resolution. Recently, we demonstrated for crystalline materials that the local transient strain field can be recorded with nanometer resolution during in situ deformation in the TEM [4,5]. Similarly, the local elastic strain in MGs can be measured by scanning nanobeam diffraction. For MGs, the strain is determined by fitting an ellipse to the first order diffraction ring. A Gatan K2 IS direct electron detector is used to acquire the diffraction patterns at a sufficient speed to perform strain mapping in situ during continuous deformation without stopping or pausing the experiment. The resulting time-resolved strain maps reveal significant heterogeneities throughout the specimen, highlighting the importance of experimental measurements of the local strain in MGs at the nanoscale. It 
is expected that the knowledge of quantitative strain fields will provide the potential for direct comparison with atomistic simulations.

References:

[1] M.F. Ashby and A.L. Greer, Scripta Materialia 54 (2006) 321.

[2] E.D. Cubuk et al., Science 358 (2017) 1033.

[3] C.A. Volkert, A. Donohue and F. Spaepen, Journal of Applied Physics 103 (2008) 83539.

[4] C. Gammer, J. Kacher, C. Czarnik, O.L. Warren, J. Ciston and A.M. Minor, Applied Physics Letters 109 (2016) 081906.

[5] T.C. Pekin, C. Gammer, J. Ciston, C. Ophus and A.M. Minor, Scripta Materialia 146 (2018) 87.

[6] The authors acknowledge support of the European Research Council under the ERC Advanced Grant INTELHYB (grant ERC-2013-ADG-340025). Work at the Molecular Foundry was supported by the Office of Science, Office of Basic Energy Sciences of the U.S. Department of Energy under Contract No. DE-AC02-05CH11231.

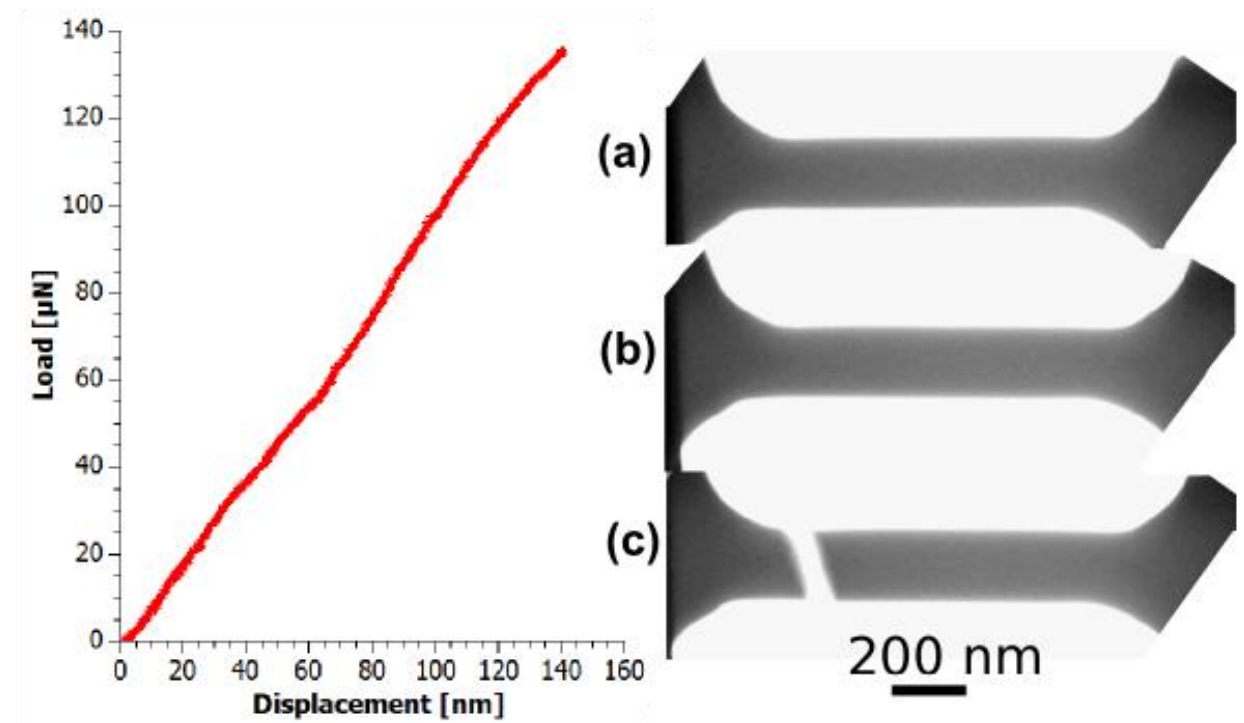

Figure 1. Load-displacement curve recorded during in situ TEM deformation of a $200 \mathrm{~nm}$ thick MG tensile specimen. The curve shows linear elastic elongation followed by sudden fracture. Two frames corresponding to the initial state and the elongated state are shown in (a) and (b), respectively. (c) After elongation the sample fractures abruptly along a single shear plane. 